\title{
Incidental Finding of Lumbar Hemangioma in a Low Back Pain Patient with Red Flag Findings
}

\author{
Amanda J Kutchman ${ }^{1}$, Jessica L Abbott ${ }^{1}$ and Lance M Mabry ${ }^{2 *}$ \\ ${ }^{1} 96^{\text {th }}$ Medical Group, Eglin Air Force Base, FL, USA \\ ${ }^{2}$ High Point University, Department of Physical Therapy, High Point, NC, USA
}

*Corresponding author: Lance M Mabry, One University Parkway, Department of Physical Therapy, High Point, NC 27262, USA

\section{ARTICLE INFO}

Received: 兆 September 22, 2021

Published: 盽 October 04, 2021

Citation: Amanda J Kutchman, Jessica L Abbott, Lance M Mabry. Incidental Finding of Lumbar Hemangioma in a Low Back Pain Patient with Red Flag Findings. Biomed J Sci \& Tech Res 39(2)-2021. BJSTR. MS.ID.006264
ABSTRACT

The views expressed in this material are those of the authors, and do not reflect the official policy or position of the U.S. Government, the Department of Defense or the Department of the Air Force. No grants or dedicated equipment was used for this study. The authors have not and will not receive any financial benefits from this study and this research has never been presented previously in any form. Incidental Finding of Lumbar Hemangioma in a Low Back Pain Patient with Red Flag Findings

Keywords: Hemangioma; Red Flag Finding; Medical Screening; Diagnostic Imaging; Magnetic Resonance Imaging; Low Back Pain; Lumbar Spine

\section{Mini Review}

The patient was a 49-year-old male Chief Master Sergeant in the Air Force with 30 years of recurrent back pain and right-sided lower extremity numbness in the S1 dermatome. His current episode of symptoms was insidious in onset 6 months prior. Since this new onset, the patient reported several red flag findings including a 16-18kg weight gain within 4 months. The patient also had episodes of bloody stools and night pain that woke him from sleeping. The patient had a known history of an L3 Schmorl's node on radiographs obtained 4 years prior (Figure 1). Aggravating factors included sitting with poor posture and elliptical use. Leaning left in a seated position and unweighting his right lower extremity eased his symptoms. Lumbar active range of motion was $25 \%$ limited in right rotation and slightly limited in flexion. Neurological screening revealed diminished sensation to light touch in the right S1 dermatome, absent right S1 deep tendon reflex, and a positive straight leg raise test.
The physical therapist referred the patient for lumbar magnetic resonance imaging due to concern his back pain was arising from sinister pathology. The MRI identified a mild L5-S1 disc protrusion and the presence of a lesion within the L1 vertebral body. The presence of fat within the lesion shown as hyperintense on T1/T2 MRI and hypointense on the Short T1 Inversion Recovery (STIR) MRI confirmed the lesion as a benign hemangioma and not a metastatic lesion (Figure 2) [1]. The diagnosis is further supported by the presence of a corduroy thickened trabecular pattern and a polka-dot trabecular pattern on axial imaging common in lumbar hemanioma (Figure 3) [2]. The patient was primarily treated using an extension based protocol. After three months of treatment, he reported "major relief" of his back pain symptoms and his range of motion was returned to normal, though the right lower extremity paresthesias remained unchanged. 

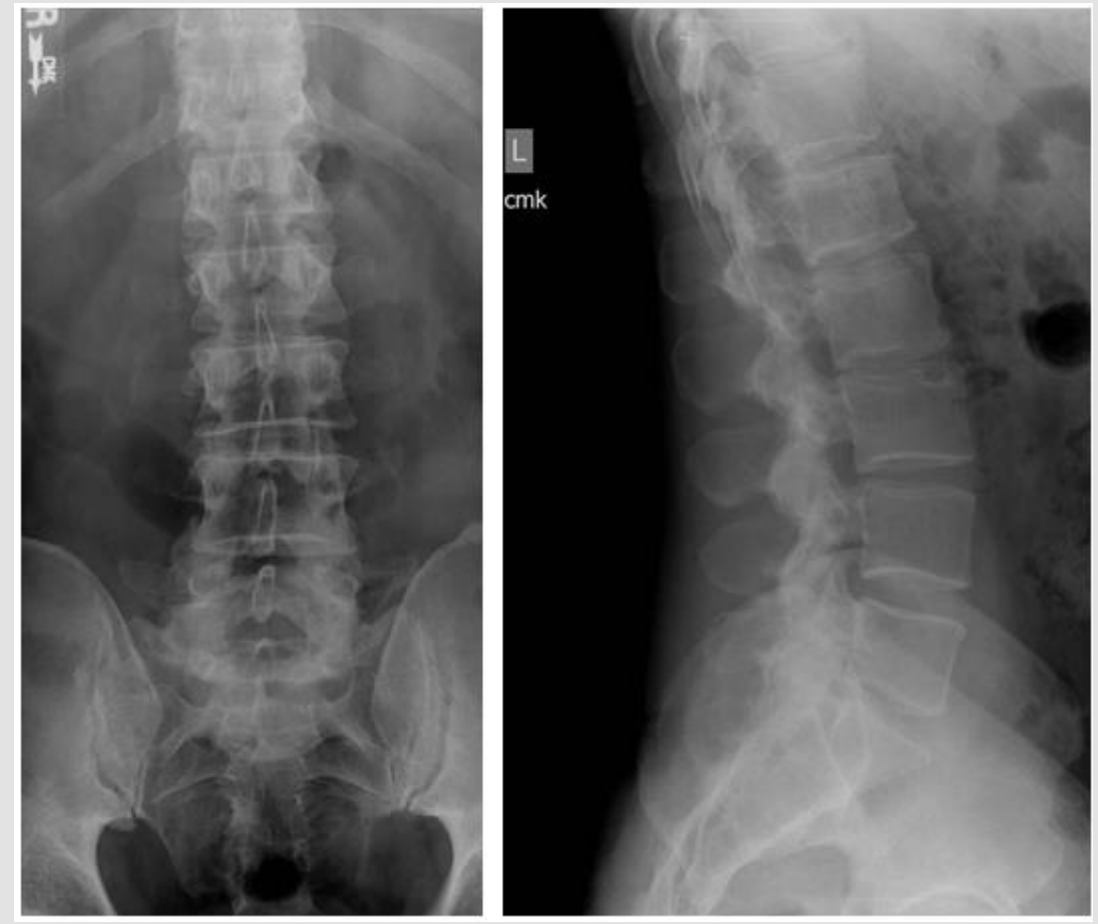

Figure 1: rom left to right:

A. Anteroposterior view and

B. Lateral view lumbar radiographs demonstrating a Schmorl's node on the superior endplate of L3.

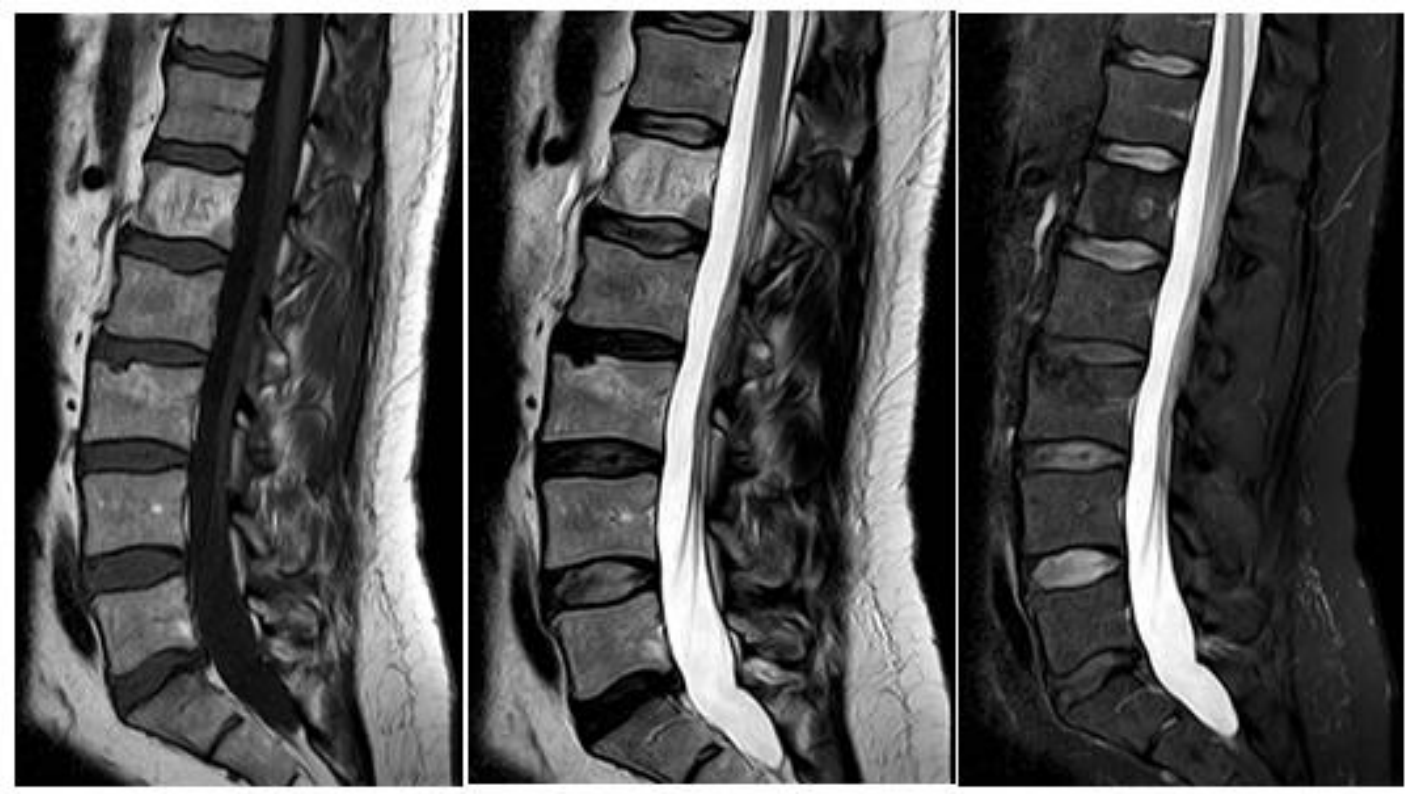

Figure 2: From left to right:

A. Sagittal T1

B. T2, and

C. Short T1 Inversion Recovery (STIR) weighted magnetic resonance images of the lumbar spine demonstrating T1/T2 hypertintensity and STIR hypointensity within the L1 vertebral body with a vertically-oriented thickened trabecular pattern consistent with lumbar hemangioma. 


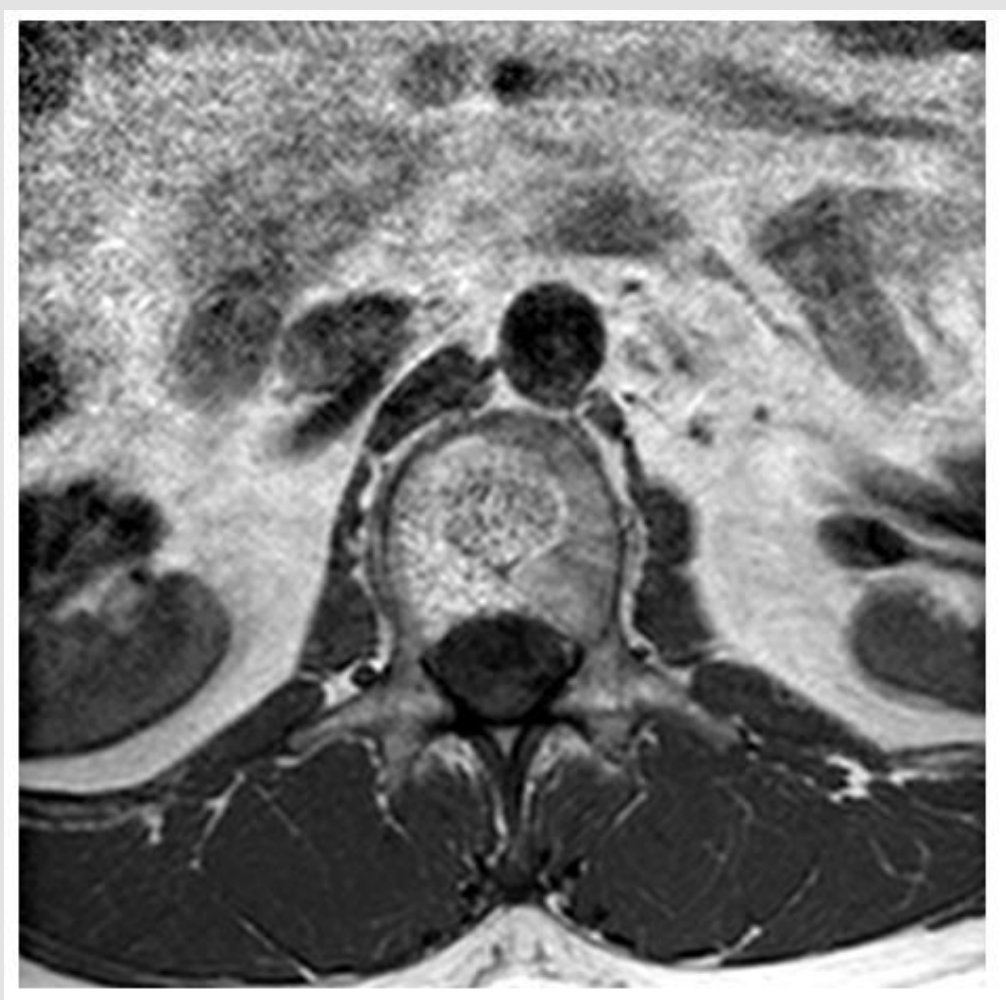

Figure 3: Axial T1 weighted magnetic resonance image of the lumbar spine demonstrating a T1 hyperintense lesion with a polka-dot appearance of the trabeculae within the L1 vertebral body consistent with lumbar hemangioma.

\section{References}

1. Park HJ, Jeon YH, Rho MH, Lee EJ, Park NH, et al. (2011) Incidental findings of the lumbar spine at MRI during herniated intervertebral disk disease evaluation. AJR American journal of roentgenology 196(5): 1151-1155.

\section{ISSN: 2574-1241}

DOI: 10.26717 /BJSTR.2021.39.006264

Lance M Mabry. Biomed J Sci \& Tech Res

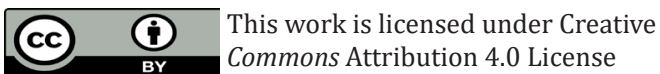

Submission Link: https://biomedres.us/submit-manuscript.php
2. Liu S-Z, Zhou X, Song A, Wang Y-P, Liu Y (2019) The corduroy appearance and the polka dot sign. QJM: An International Journal of Medicine 113(3): 222-223.

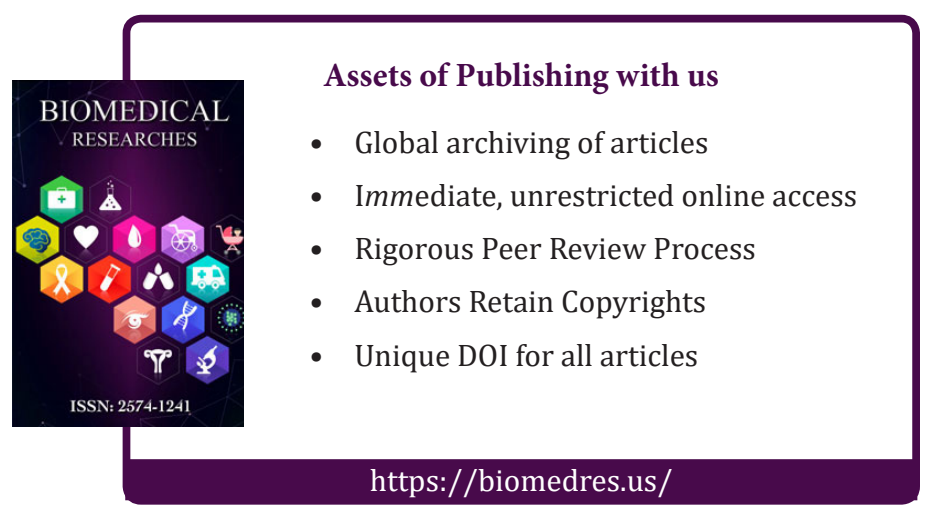

